\title{
Atlas of neonatal face images using triangular meshes
}

\author{
Pedro A. S. S. Orona*, Davi A. D. Fabbro*, Tatiany M. Heiderich ${ }^{\dagger}$, Marina C. M. Barros ${ }^{\dagger}$, \\ Rita C. X. Balda ${ }^{\dagger}$, Ruth Guinsburg ${ }^{\dagger}$, Carlos E. Thomaz \\ *Departamento de Engenharia Elétrica, FEI. São Bernardo do Campo, São Paulo \\ ${ }^{\dagger}$ Departamento de Medicina Neonatal, Universidade Federal de São Paulo. São Paulo, São Paulo
}

\begin{abstract}
Resumo-Over the last years, neonatal face analysis has allowed the investigation and creation of non-invasive methods that enable classification of painful stimulus in newborns. In this context, changes on facial movements and expression have provided relevant scientific information and clinical significance, since they describe the presence of the pain itself perceived by the newborns. In this work, we propose and implement a computational framework that uses triangular meshes, with the goal of generating high resolution spatially normalized atlases potentially useful for automatic neonatal pain assessment.

Index Terms-registration, atlas, pain
\end{abstract}

\section{INTRODUÇÃO}

A dor é uma característica inerente à vida em si, expressa em todos os organismos vivos que possuem sistema nervoso central [1] e serve para indicar que algo está errado. É um alerta que gera incômodo, uma experiência emocionalmente ruim e evitada conscientemente, apesar de ser essencial para nossa sobrevivência, pois sinaliza a presença de dano corporal ou ameaça de morte, promovendo um comportamento de fuga [2]. Devido a essas características, há séculos, o alívio da dor tem sido tópico de estudos, desde tratamentos elétricos até a evolução dos tratamentos farmacológicos com derivados opioides, como morfina, e outros analgésicos [3].

Para seres humanos adultos, a dor pode ser facilmente identificada por meio de expressões e indicações objetivas, e tal identificação somente não é possível quando o indivíduo se encontra inconsciente, sendo necessária a aplicação de técnicas subjetivas [4]. Em lactentes pré-verbais, entre os quais se incluem os recém-nascidos, existe, no entanto, uma dificuldade maior em identificar a dor, principalmente em quais níveis esta se manifesta, devido à impossibilidade de comunicação verbal direta e objetiva. Nestas situações, a identificação da dor se dá por meio da análise de ações e reações, não necessariamente objetivas, do recém-nascido frente ao estímulo desagradável, potencial causa de dor [4]. Tal dificuldade se dá em um contexto bastante adverso, pois o recém-nascido, dada a sua imaturidade neurológica, é mais sensível e susceptível a experiências ruins como dor e estresse, que podem trazer complicações no desenvolvimento a curto e médio prazos, com impacto negativo na evolução neurológica, cognitiva e psiquiátrica deste indivíduo [5]-[8].

Recentemente, tem havido um interesse crescente em compreender as respostas comportamentais relacionadas à dor baseadas, principalmente, em expressões faciais $[\overline{8}]-[16]$. A análise automática de dor em imagens faciais é um tópico emergente em inteligência artificial dado o aumento de demanda por monitoramentos de dor contínuos e consistentes em clínicas e centros de saúde de recém-nascidos. Esse aumento considerável no interesse pela utilização de métodos de aprendizagem de máquina e visão computacional com o intuito de analisar expressões faciais de dor em recém-nascidos se dá pelo fato de que das várias modalidades de expressão não verbal (por exemplo, movimento corporal, vocalizações), a atividade facial pode fornecer a informação mais sensível, específica e acessível sobre a presença, natureza e gravidade da dor ao longo da vida, desde a infância [17] até a idade avançada [18].

No entanto, diferenças de iluminação e também variações posicionais dos bebês no momento da captura das imagens não são obviamente discriminantes para o fenômeno dor, e acabam atrapalhando a análise computacional posterior. Isto dado o fato de que a maioria dos trabalhos nesta área foram desenvolvidos utilizando modelos de face que se baseiam em diferença da distância entre pontos fiduciais da face, ou seja, é extremamente necessário que os dados originais sejam normalizados espacialmente, garantindo que haja uma correspondência no momento da comparação entre esses pontos.

A principal contribuição deste trabalho é descrever e implementar uma metodologia de normalização espacial de imagens faciais de recém-nascidos por meio de uma estrutura bem definida de passos computacionais. Mais especificamente, propomos e implementamos um framework computacional que combina posicionamento de pontos de referências (landkmarks) faciais e registro não-rígido de imagens, utilizando funções triangulares. Para a construção do atlas de imagens de faces de recém-nascidos, o framework proposto baseiase em trabalhos multidisciplinares anteriores aplicados para combinação de faces [19]-[21], registro de imagens médicas [22], construção de atlas cerebral [23], [24] e morfologia facial $[25]$.

As seções restantes deste artigo são descritas a seguir. Apresentamos na seção II uma breve descrição das bases de imagens de face de recém-nascidos utilizadas aqui. Na seção III. explicamos o framework proposto para a construção do atlas das imagens faciais e para extração de características de dor a serem utilizadas na análise multivariada. Todos os experimentos realizados e seus correspondentes resultados são apresentados na seção[IV] Por fim, apresentamos as conclusões 


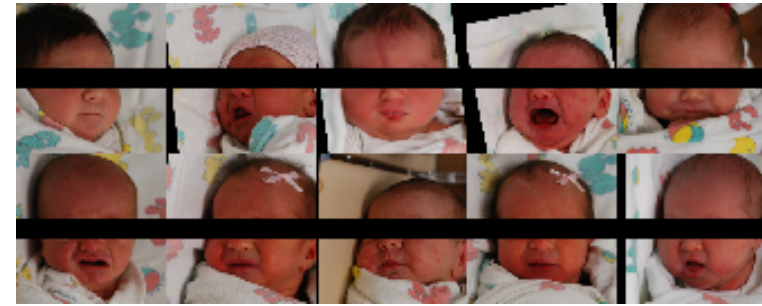

Figura 1: Exemplos da base de dados COPE.

finais deste trabalho na seção $\mathrm{V}$

\section{BASE DE DADOS}

\section{A. COPE}

Este conjunto de dados consiste em 200 imagens coloridas capturadas de 26 bebês caucasianos, 50\% meninas, usando uma câmera digital Nikon D100 [9]. A faixa etária dos bebês varia de dezoito horas a três dias e todos os bebês estavam em bom estado de saúde. As imagens faciais das crianças foram capturadas enquanto estas eram submetidas a quatro estímulos diferentes: estímulo da dor durante a punção do calcanhar (60 imagens), estímulo de descanso / choro durante o transporte de uma criança de um berço para outro (63 imagens de descanso e 18 imagens de choro), estímulo de ar no nariz (23 imagens) e o estímulo de fricção, que envolve realizar atrito na superfície lateral externa do calcanhar com algodão embebido em álcool (36 imagens). Além desses quatro estímulos, o banco de dados inclui imagens dos bebês em estado neutro de descanso.

A Figura 1 apresenta um conjunto de exemplos da base de dados COPE.

\section{B. UNIFESP}

O banco de imagens da UNIFESP foi desenvolvido durante uma pesquisa de doutorado [8], cujo objetivo era a construção de um software capaz de identificar automaticamente a expressão de dor em recém-nascidos através de imagens bidimensionais capturadas.

A base foi construída a partir de fotos capturadas antes, durante e depois do procedimento aplicado aos recém-nascidos, podendo esses procedimentos ser uma punção venosa, capilar ou injeção intramuscular, procedimentos comuns e necessários, porém considerando a fragilidade do recém-nascido são procedimentos invasivos, dolorosos e/ou estressantes. A captura dessas fotos foi feita a cada 3 segundos por um aparato composto por três câmeras posicionadas ao redor do berço [8].

Entre as imagens capturadas pelo sistema, foram escolhidas 12 imagens faciais de cada um dos 30 recém-nascidos, de maneira aleatória, totalizando 360 imagens separadas, onde 138 foram adquiridas antes do procedimento, 30 durante o procedimento e 192 até dez minutos após o procedimento.

A Figura 2 apresenta um conjunto de exemplos da base de dados UNIFESP.

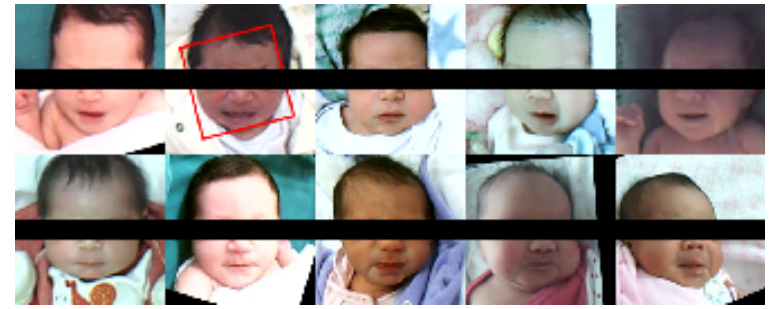

Figura 2: Exemplos da base de dados UNIFESP.

\section{CONSTRUÇÃo DO ATLAS}

\section{A. Pre-processamento}

A primeira etapa para construção do atlas é o préprocessamento das imagens das bases COPE e UNIFESP. Esta etapa consiste em remover as características que dificultam a realização das próximas etapas, como orientação das imagens, quantidade diferente de pixels e erros na forma como as imagens foram capturadas.

Para a base COPE, o primeiro pré-processamento realizado foi a redução na quantidade de pixels de $3000 \times 2000$ para $752 \times 500$ (quatro vezes menor), com o objetivo de otimizar o consumo de memória e tempo de processamento das etapas subsequentes. Em seguida foi observado que as imagens de face não estavam orientadas verticalmente, o que degrada a qualidade da predição dos pontos fiduciais da face. Portanto, as imagens foram rotacionadas em até $90^{\circ}$ ou $-90^{\circ}$, dependendo da orientação da imagem.

Para a base UNIFESP, os 130 pixels à direita de todas as imagens são brancos (valor 255 na representação de 8 bits), o que permite que esta informação da imagem possa ser removida, resultando em imagens com 320 pixels de largura. Além disso, as imagens da base UNIFESP não apresentam uniformidade na quantidade de pixels na vertical (algumas contém 233 e outras 221 pixels), portanto as imagens com 233 pixels de altura foram reduzidas para 221 pixels. O tamanho final das imagens da UNIFESP pré-processadas ficou padronizado em $320 \times 221$.

\section{B. Predição dos pontos fiduciais da face}

Neste trabalho, o alinhamento das imagens é realizado exclusivamente pelas informações geométricas das imagens, ou seja, a informação de textura não é utilizada. Desta forma, a informação utilizada são os pontos fiduciais da face.

A predição desses pontos é feita por um algoritmo baseado em deep learning, disponível publicamente ${ }^{1}$ [26], que por sua vez utiliza uma rede convolucional para detecção de faces [27], e em seguida marca 68 pontos fiduciais, que podem ser visualizados pelos pixels brancos da Figura 3(a).

\section{Alinhamento global}

A transformação utilizada para alinhamento global é também chamada de transformação afim por similaridade, que permite translação, rotação e escala e tem a propriedade de

${ }^{1}$ https://github.com/1adrianb/face-alignment 
manter as proporções da imagem. Esta é uma transformação linear, ou seja, a mesma transformação é aplicada sobre todos os pixels. A transformação é definida por uma matriz:

$$
\mathbf{T}=\left[\begin{array}{ccc}
\lambda \cdot \cos (\theta) & \lambda \cdot \operatorname{sen}(\theta) & t_{x} \\
-\lambda \cdot \operatorname{sen}(\theta) & \lambda \cdot \cos (\theta) & t_{y} \\
0 & 0 & 1
\end{array}\right]
$$

onde $\lambda$ é o fator de escala, $\theta$ o ângulo de rotação, e $t_{x}$ e $t_{y}$, são as translações horizontais e verticais, respectivamente [28].

Dos 68 pontos fiduciais, 5 pontos foram escolhidos para o alinhamento global. Estes cinco pontos representam regiões de interesse importantes da face, como centro dos olhos, nariz e cantos da boca.

Considerando $\mathbf{u}$ as coordenadas $x$ e $y$ dos 5 pontos iniciais (pontos de saída) e $\mathbf{u}^{\prime}$ as coordenadas $x$ e $y$ dos 5 pontos da imagem alinhada globalmente (pontos de destino), é possível descrever a equação:

$$
\mathbf{u}^{\prime}=\mathbf{T} \cdot \mathbf{u}
$$

onde os parâmetros da matriz $\mathbf{T}$ podem ser estimados ao formular esta equação como um problema de mínimos quadrados [28].

As etapas do alinhamento global podem ser visualizadas na Figura 3 . (a) Original preprocessada

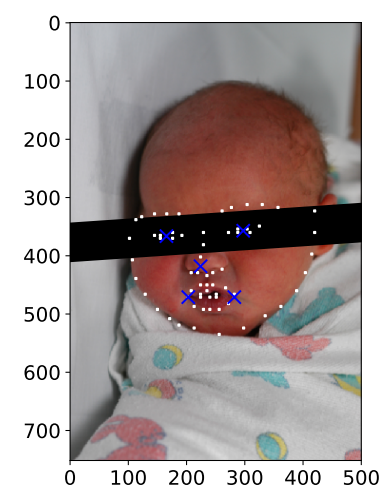

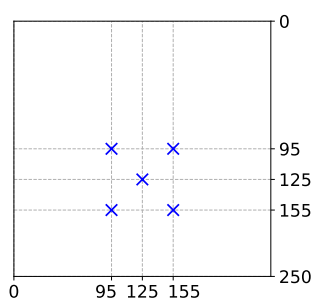

(b) Referencia

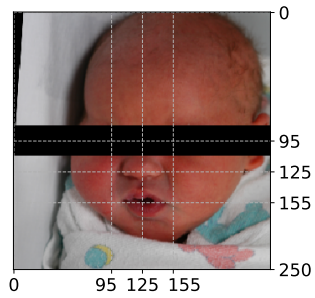

(c) Alinhada
Figura 3: Etapas para normalização global das imagens: (a) marcação automática dos 68 pontos fiduciais na imagem original preprocessada, onde os 5 pontos correspondentes aos olhos, nariz e cantos da boca estão representados por um ' $x$ ' em azul, (b) especificação das 5 coordenadas de destino e (c) a imagem normalizada globalmente.

\section{Normalização não-rígida}

O método de normalização não-rígida adotado neste trabalho é baseado em malhas triangulare ${ }^{2}$ que já foi utilizado para

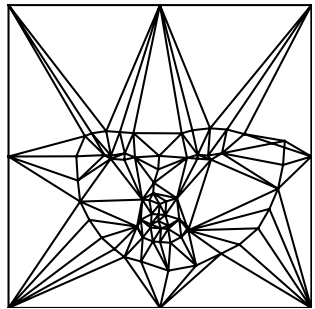

(a) Malha de saída

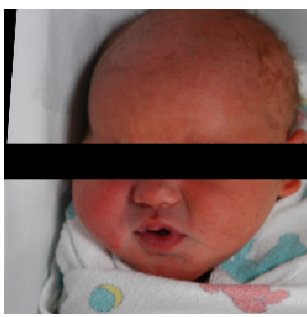

(c) Registro global

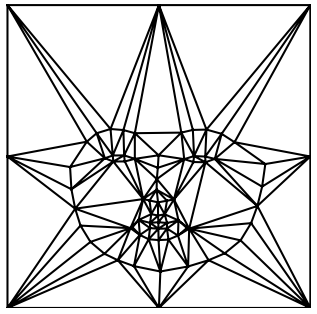

(b) Malha de destino

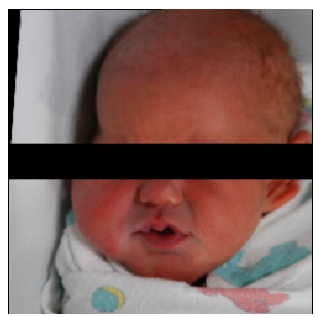

(d) Registro não rígido
Figura 4: Etapas para normalização não-rígida das imagens: (a) especificação da malha triangular de saída calculada a partir dos 68 pontos fiduciais da imagem a ser deformada, (b) especificação da malha triangular de destino calculada a partir da média dos 68 pontos fiduciais das imagens normalizadas globalmente, (c) a imagem correspondente à malha de saída e (d) a imagem resultante normalizada não rigidamente.

alinhamento de faces em artigos anteriores de reconhecimento facial [29].

Com as imagens globalmente alinhadas, é possível encontrar os 68 pontos fiduciais médios para cada base de dados, que por sua vez serão utilizados para calcular uma malha triangular por meio do algoritmo de triangulação de Delaunay [30]. A malha triangular formada pelos 68 pontos fiduciais é chamada neste trabalho de malha de destino.

Em seguida, para os 68 pontos fiduciais de cada imagem, computa-se uma malha triangular com base nos vértices da malha de destino para garantir que a quantidade de triângulos é a mesma. Esta malha especifica para cada imagem é chamada neste trabalho de malha de saída.

Finalmente, para cada triângulo da malha de saída foi calculada uma transformação afim capaz de deformar exatamente para a forma do triângulo correspondente à malha de destino.

A transformação afim de cada triângulo é calculada de forma similar ao método descrito na seção anterior, com a diferença de que a estimação dos parâmetros da transformação afim é feita com base em 3 pontos, que correspondem aos vértices de cada triângulo, portanto existe uma solução única para deformação de cada triângulo [28].

Adicionalmente, para manter a informação fora da região formada pelos 68 pontos fiduciais, foram acrescentados 8 pontos nas extremidades de cada imagem, resultando, por exemplo, as imagens da Figura 4

\footnotetext{
${ }^{2}$ Conhecido em inglês por piecewise
} 


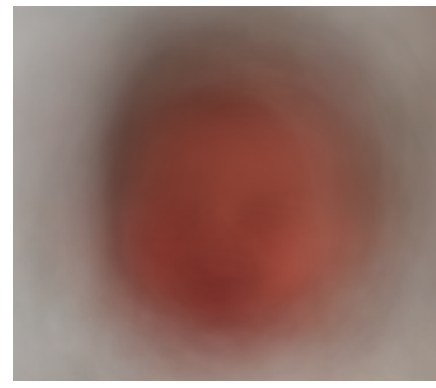

(a)

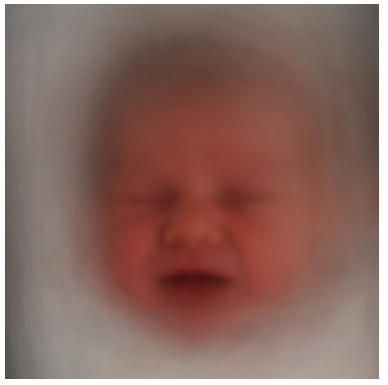

(b)

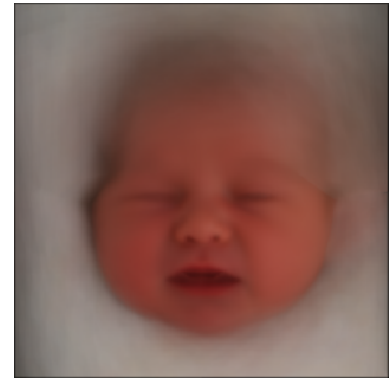

(c)

Figura 5: Atlas das imagens da COPE: (a) original, (b) normalizadas globalmente e (c) normalizadas não rigidamente.

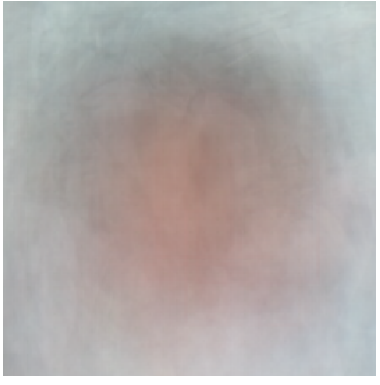

(a)

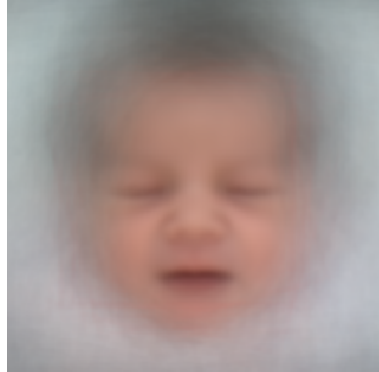

(b)

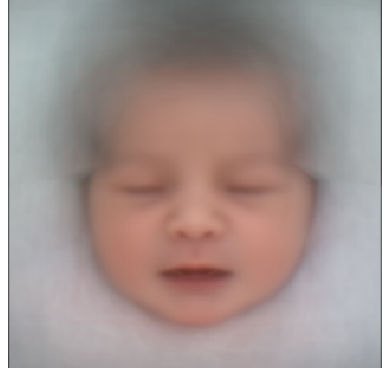

(c)

Figura 6: Atlas das imagens da UNIFESP: (a) original, (b) normalizadas globalmente e (c) normalizadas não rigidamente.

\section{Resultados}

Utilizando os métodos descritos acima, foram gerados os atlas de imagens de faces de recém-nascidos através do cálculo da média. Para a base de dados COPE apresentamos as imagens médias sem nenhum tipo de normalização aplicada às imagens na Figura 5a, normalizadas globalmente na Figura $5 b$, e normalizadas não rigidamente na Figura $5 \mathrm{c}$ Por meio da mesma metodologia, obtemos os atlas para o banco de imagens desenvolvido pela UNIFESP, mostradas analogamente nas Figuras 6a, 6b ec.

Vemos claramente a evolução alcançada por meio do método proposto, dada a alta resolução dos detalhes faciais apresentados no atlas das imagens geradas, primeiramente, pela normalização global e, por fim, pela não-rígida. Esta última nos proporciona uma imagem de alta definição e colorida, a qual pode ser utilizada como referência em trabalhos que desejem normalizar as bases apresentadas e que não possuem em mãos um atlas de alta resolução.

Com o intuito de se verificar e avaliar os resultados obtidos em relação aos atlas global e não-rígido, estes foram comparados qualitativamente através de imagens de desvio padrão (erro), além de métricas quantitativas de similaridade.

\section{A. Imagens de erro}

Como visto anteriormente, para os atlas das imagens, ocorre uma melhor definição dos detalhes faciais, além do contorno da face, e isto fica evidenciado nas Figuras 7 e 8 , onde temos valores muito maiores de desvio principalmente em relação ao contorno comparando-se a normalização global e a nãorígida. As variações são ainda maiores quando comparamos os resultados obtidos pelas normalizações e o atlas das imagens originais.

Especificamente, analisando a Figura 7c, vemos ainda valores diferentes de zero de desvio padrão em detalhes da face como olhos e narinas, e acreditamos que isso se dá por alguns fatores. Primeiramente, através de uma rápida exploração, podemos comprovar que existem amostras dentro da base COPE que estão de olhos abertos e outras de olhos fechados, e isto é capturado pelo cálculo do desvio pois a normalização realizada irá apenas ajustar a amostra baseada em seus pontos fiduciais, ou seja, um olho aberto na imagem original, permanecerá aberto após a normalização, e viceversa. Para o caso das narinas, temos amostras em ângulos que não chegam a ilustrar as narinas, aonde a câmera se posicionou um pouco acima do bebê, e outras aonde as narinas estão muito expostas, para o caso em que a câmera adquiriu uma imagem mais abaixo da face do bebê. Portanto, dadas essas diferenças, semelhante ao que ocorre com os olhos, isto fica evidenciado na imagem do desvio padrão, em que as narinas possuem valores de desvio maiores em relação aos outros detalhes da face.

Adicionalmente, analisando a Figura $8 \mathrm{c}$ e tendo em mente que a base desenvolvida pela UNIFESP também apresenta as mesmas variações de angulação de câmera e estado dos olhos, podemos chegar às mesmas conclusões obtidas anteriormente para o desvio padrão do atlas das imagens normalizadas não rigidamente. 


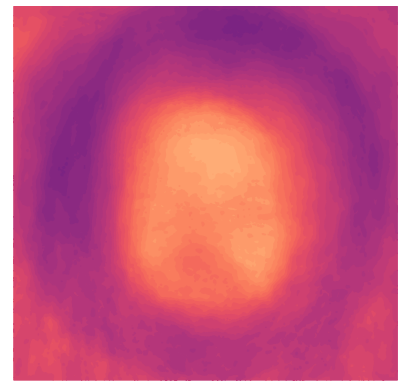

(a)

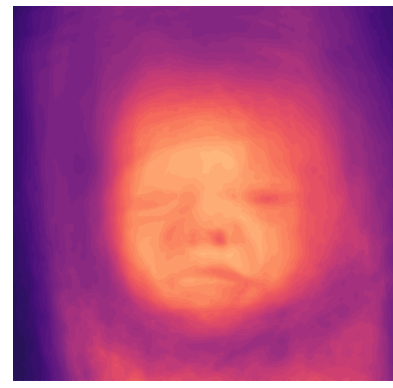

(b)

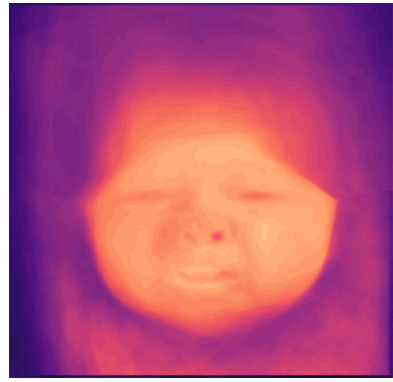

(c)

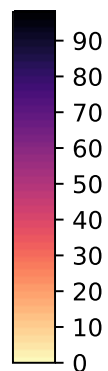

(d)

Figura 7: Desvio padrão dos atlas das imagens da COPE: (a) original, (b) normalizadas globalmente e (c) normalizadas não rigidamente

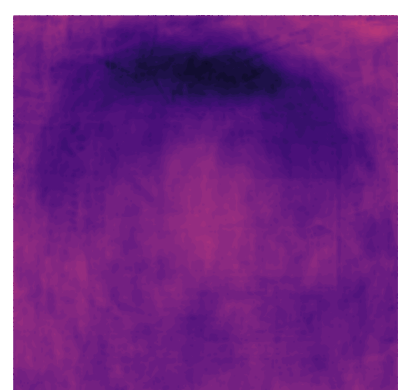

(a)

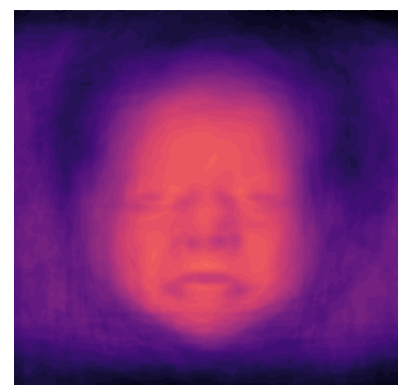

(b)

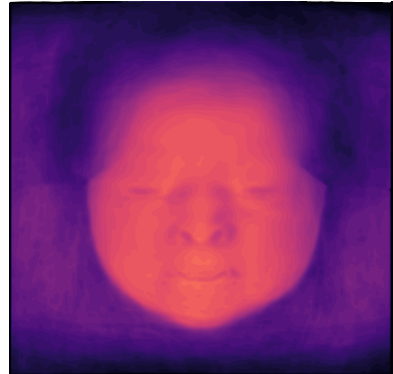

(c)

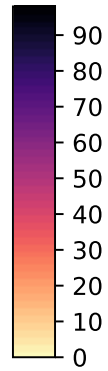

(d)

Figura 8: Desvio padrão dos atlas das imagens da UNIFESP: (a) original, (b) normalizadas globalmente e (c) normalizadas não rigidamente

\section{B. Métricas de Similaridade}

Além da análise qualitativa das imagens geradas pelo cálculo do desvio padrão, podemos fazer uso de métricas de similaridade que nos oferecem um resultado quantitativo para a avaliação dos atlas.

Para o cálculo dessas métricas, a área de interesse foi definida pela envoltória convexa formada pelos 68 pontos fiduciais.

Essas métricas, comumente utilizadas na literatura, são: MSE (Mean Squared Error), PCC (Pearson Correlation Coefficient), NMI (Normalized Mutual Information) e $\Sigma L^{2}$ (Norma $L^{2}$ ) [31].

1) Erro quadrático médio:

$$
M S E=\frac{1}{N} \sum_{i=1}^{N}\left(I_{M O V_{i}}-I_{R E F_{i}}\right)^{2} .
$$

2) Coeficiente de Correlação de Pearson:

$$
P_{C C}=\frac{\sum_{i=1}^{N}\left(I_{M O V_{i}}-\overline{\mathbf{I}_{M O V}}\right)\left(I_{R E F_{i}}-\overline{\mathbf{I}_{R E F}}\right)}{\sqrt{\sum_{i=1}^{N}\left(I_{M O V_{i}}-\overline{\mathbf{I}_{M O V}}\right)^{2} \sum_{i=1}^{N}\left(I_{R E F_{i}}-\overline{\mathbf{I}_{R E F}}\right)^{2}}} .
$$

3) Informação Mútua Normalizada:

$$
N M I=\frac{H\left(\mathbf{I}_{M O V}\right)+H\left(\mathbf{I}_{R E F}\right)}{H\left(\mathbf{I}_{M O V}, \mathbf{I}_{R E F}\right)},
$$

4) Norma $L^{2}$ :

$\Sigma L^{2}=\sum_{i=1}^{n} \sqrt{\left(x_{M O V_{i}}-x_{R E F_{i}}\right)^{2}+\left(y_{M O V_{i}}-y_{R E F_{i}}\right)^{2}}$.

\begin{tabular}{|c||c|c|c|}
\hline \multicolumn{4}{|c|}{ COPE — Métricas de similaridade } \\
\hline & Original & Global & Não-rígida \\
\hline \hline MSE $(\downarrow)$ & $639 \pm 268$ & $748 \pm 329$ & $\mathbf{1 2 9} \pm \mathbf{8 7}$ \\
PCC $(\uparrow)$ & $0.73 \pm 0.08$ & $0.79 \pm 0.09$ & $\mathbf{0 . 9 7} \pm \mathbf{0 . 0 2}$ \\
NMI $(\uparrow)$ & $0.44 \pm 0.05$ & $0.57 \pm 0.05$ & $\mathbf{0 . 6 8} \pm \mathbf{0 . 0 1}$ \\
$\Sigma L^{2}(\downarrow)$ & $2668 \pm 1099$ & $519 \pm 215$ & $\mathbf{0 . 0 0} \pm \mathbf{0 . 0 0}$ \\
\hline
\end{tabular}

Tabela I: Semelhança entre as imagens da base COPE.

\begin{tabular}{|c||c|c|c|}
\hline \multicolumn{4}{|c|}{ UNIFESP - Métricas de similaridade } \\
\hline & Original & Global & Não-rígida \\
\hline \hline MSE $(\downarrow)$ & $2104 \pm 856$ & $1147 \pm 597$ & $\mathbf{3 7 9} \pm \mathbf{2 4 0}$ \\
PCC $(\uparrow)$ & $0.43 \pm 0.21$ & $0.82 \pm 0.07$ & $\mathbf{0 . 9 7} \pm \mathbf{0 . 0 2}$ \\
NMI $(\uparrow)$ & $0.33 \pm 0.14$ & $0.59 \pm 0.05$ & $\mathbf{0 . 7 0} \pm \mathbf{0 . 0 1}$ \\
$\Sigma L^{2}(\downarrow)$ & $2776 \pm 1175$ & $464 \pm 163$ & $\mathbf{0 . 0 0} \pm \mathbf{0 . 0 0}$ \\
\hline
\end{tabular}

Tabela II: Semelhança entre as imagens da base da UNIFESP.

As Tabelas [e $\mathrm{I}$ nos fornecem mais uma comparação entre os atlas gerados para cada base de imagens. A normalização não-rígida obteve, para ambos os conjuntos de dados, COPE e UNIFESP, os melhores resultados em todas as métricas de similaridade utilizadas. Vale destacar o valor 0 alcançado 
com a normalização proposta utilizando a norma $L^{2}$, como era de se esperar, pois o processo de triangulação força $o$ reposicionamento dos pontos fiduciais de maneira que estes estejam exatamente na mesma posição quando comparada a imagem a ser normalizada com a de referência.

\section{Conclus Ão}

Atlas são necessários para representar tanto informações espaciais quanto conceituais de um determinado objeto de interesse. Para o problema em questão, essas formas de representação de imagens de referência são essenciais para descrever um padrão de face tão característico e detalhado quanto possível para recém-nascidos, evitando que efeitos ou sinais da imagem que não sejam relevantes e que retratem particularidades indesejadas inerentes a todo processo imperfeito de aquisição de dados não sejam propagados erroneamente como variações discriminantes.

Este trabalho propõe e implementa um framework computacional para construção de atlas de imagens de face de recémnascidos. O framework, como apresentado, produz modelos realistas de face não-enviesados com um alto grau de detalhamento. Todos os resultados apresentados podem ser reproduzidos utilizando os códigos disponibilizados publicamente ${ }^{3}$

O presente trabalho foi realizado com apoio da Coordenação de Aperfeiçoamento de Pessoal de Nível Superior - Brasil (CAPES) - Código de Financiamento 001.

\section{REFERÊNCIAS}

[1] K. J. Anand and K. D. Craig, "New perspectives on the definition of pain," Pain-Journal of the International Association for the Study of Pain, vol. 67, no. 1, pp. 3-6, 1996.

[2] Luda, A. G. Nackley, I. E. Tchivileva, S. A. Shabalina, and W. Maixner, "Genetic architecture of human pain perception," TRENDS in Genetics, vol. 23, no. 12, pp. 605-613, 2007.

[3] M. Zimmermann, "Geschichte der schmerztherapie 1500 bis 1900," Der Schmerz, vol. 21, no. 4, pp. 297-306, 2007.

[4] R. Guinsburg and M. Cuenca, "A linguagem da dor no recém-nascido. documento científico do departamento de neonatologia sociedade brasileira de pediatria: São paulo, 8 de outubro de 2010 [cited 2014 oct 20]," 2010.

[5] R. Guinsburg, "Avaliação e tratamento da dor no recém-nascido," $J$ Pediatr (Rio J), vol. 75, no. 3, pp. 149-60, 1999.

[6] A. G. Chermont, R. Guinsburg, R. d. C. X. Balda, and B. I. Kopelman, "O que os pediatras conhecem sobre avaliação e tratamento da dor no recém-nascido?" Jornal de Pediatria, 2003.

[7] R. E. Grunau, "Neonatal pain in very preterm infants: long-term effects on brain, neurodevelopment and pain reactivity," Rambam Maimonides medical journal, vol. 4, no. 4, 2013.

[8] T. M. Heiderich, A. T. F. S. Leslie, and R. Guinsburg, "Neonatal procedural pain can be assessed by computer software that has good sensitivity and specificity to detect facial movements," Acta Paediatrica, vol. 104, no. 2, pp. e63-e69, 2015.

[9] S. Brahnam, C.-F. Chuang, F. Y. Shih, and M. R. Slack, "Machine recognition and representation of neonatal facial displays of acute pain," Artificial intelligence in medicine, vol. 36, no. 3, pp. 211-222, 2006.

[10] S. Brahnam, C.-F. Chuang, R. S. Sexton, and F. Y. Shih, "Machine assessment of neonatal facial expressions of acute pain," Decision Support Systems, vol. 43, no. 4, pp. 1242-1254, 2007.

[11] B. Gholami, W. M. Haddad, and A. R. Tannenbaum, "Relevance vector machine learning for neonate pain intensity assessment using digital imaging," IEEE Transactions on biomedical engineering, vol. 57, no. 6, pp. 1457-1466, 2010.
[12] L. Nanni, S. Brahnam, and A. Lumini, "A local approach based on a local binary patterns variant texture descriptor for classifying pain states," Expert Systems with Applications, vol. 37, no. 12, pp. 78887894, 2010.

[13] M. N. Mansor and M. N. Rejab, "Infant pain recognition system with glcm features and gann under unstructed lighting condition," in Control System, Computing and Engineering (ICCSCE), 2013 IEEE International Conference on. IEEE, 2013, pp. 243-248.

[14] G. Lu, C. Yang, M. Chen, and X. Li, "Sparse representation based facial expression classification for pain assessment in neonates," in Natural Computation, Fuzzy Systems and Knowledge Discovery (ICNC-FSKD), 2016 12th International Conference on. IEEE, 2016, pp. 1615-1619.

[15] G. Zamzmi, D. Goldgof, R. Kasturi, and Y. Sun, "Neonatal pain expression recognition using transfer learning," arXiv preprint arXiv:1807.01631, 2018.

[16] R. Zhi, G. Zamzmi, D. Goldgof, T. Ashmeade, and Y. Sun, "Automatic infants' pain assessment by dynamic facial representation: Effects of profile view, gestational age, gender, and race," Journal of clinical medicine, vol. 7, no. 7, p. 173, 2018.

[17] R. V. Grunau and K. D. Craig, "Pain expression in neonates: facial action and cry," Pain, vol. 28, no. 3, pp. 395-410, 1987.

[18] T. Hadjistavropoulos, K. Herr, K. M. Prkachin, K. D. Craig, S. J. Gibson, A. Lukas, and J. H. Smith, "Pain assessment in elderly adults with dementia," The Lancet Neurology, vol. 13, no. 12, pp. 1216-1227, 2014.

[19] S. J. Gibson, "Eigenfit: A statistical learning approach to facial composites," Ph.D. dissertation, University of Kent, 2006.

[20] I. Xavier, M. Pereira, G. Giraldi, S. Gibson, C. Solomon, D. Rueckert, D. Gillies, and C. Thomaz, "A photo-realistic generator of most expressive and discriminant changes in 2d face images," in 2015 Sixth International Conference on Emerging Security Technologies (EST). IEEE, 2015, pp. 80-85.

[21] I. Xavier, G. Giraldi, S. Gibson, G. Gattas, D. Rueckert, and C. Thomaz, "Construction of a spatio-temporal face atlas: Experiments using down syndrome samples," in Conference on Graphics, Patterns and Images (SIBGRAPI): Workshop on Face Processing Applications, 2016, pp. 14.

[22] D. Rueckert, L. I. Sonoda, C. Hayes, D. L. Hill, M. O. Leach, and D. J. Hawkes, "Nonrigid registration using free-form deformations: application to breast mr images," IEEE transactions on medical imaging, vol. 18 , no. 8, pp. 712-721, 1999.

[23] A. Serag, P. Aljabar, G. Ball, S. J. Counsell, J. P. Boardman, M. A. Rutherford, A. D. Edwards, J. V. Hajnal, and D. Rueckert, "Construction of a consistent high-definition spatio-temporal atlas of the developing brain using adaptive kernel regression," Neurolmage, vol. 59, no. 3, pp. $2255-2265,2012$.

[24] P. Aljabar, K. K. Bhatia, M. Murgasova, J. V. Hajnal, J. P. Boardman, L. Srinivasan, M. A. Rutherford, L. Dyet, A. D. Edwards, and D. Rueckert, "Assessment of brain growth in early childhood using deformationbased morphometry," Neuroimage, vol. 39, no. 1, pp. 348-358, 2008.

[25] J. R. Shaffer, E. Orlova, M. K. Lee, E. J. Leslie, Z. D. Raffensperger, C. L. Heike, M. L. Cunningham, J. T. Hecht, C. H. Kau, N. L. Nidey et al., "Genome-wide association study reveals multiple loci influencing normal human facial morphology," PLoS genetics, vol. 12, no. 8, p. e1006149, 2016.

[26] A. Bulat and G. Tzimiropoulos, "How far are we from solving the $2 \mathrm{~d} \& 3 \mathrm{~d}$ face alignment problem? (and a dataset of 230,000 3d facial landmarks)," in International Conference on Computer Vision, 2017.

[27] S. Zhang, X. Zhu, Z. Lei, H. Shi, X. Wang, and S. Z. Li, "S3fd: Single shot scale-invariant face detector," in Proceedings of the IEEE International Conference on Computer Vision, 2017, pp. 192-201.

[28] S. Umeyama, "Least-squares estimation of transformation parameters between two point patterns," IEEE Transactions on Pattern Analysis \& Machine Intelligence, no. 4, pp. 376-380, 1991.

[29] Y. Taigman, M. Yang, M. Ranzato, and L. Wolf, "Deepface: Closing the gap to human-level performance in face verification," in Proceedings of the IEEE conference on computer vision and pattern recognition, 2014, pp. 1701-1708.

[30] C. B. Barber, D. P. Dobkin, and H. Huhdanpaa, "The quickhull algorithm for convex hulls," ACM TRANSACTIONS ON MATHEMATICAL SOFTWARE, vol. 22, no. 4, pp. 469-483, 1996.

[31] J. A. Maintz and M. A. Viergever, "A survey of medical image registration," Medical image analysis, vol. 2, no. 1, pp. 1-36, 1998.

${ }^{3}$ https://github.com/IPL-FEI/newborn-pain-atlas 